\title{
Low-molecular-weight S-nitrosothiols and blood vessel injury
}

\author{
Philip A. Marsden \\ Renal Division and Department of Medicine, St. Michael's Hospital and University of Toronto, Toronto, Ontario, Canada.
}

\begin{abstract}
$S$-nitrosothiol signaling reactions are argued to play key modulatory roles in mediating the actions of NOS in health and disease. A report by Palmer et al. in this issue of the JCI provides new insight into the in vivo biology of $S$-nitrosothiols (see the related article beginning on page 2592). The authors examine the chronic effects of exogenous nitrosothiol therapy and demonstrate that the commonly used antioxidant $N$-acetylcysteine (NAC) induces pulmonary arterial hypertension in mice. Importantly, the authors argue that the vascular pathology they observe in the lungs of these animals is functionally and morphologically equivalent to that observed in chronic hypoxia. These findings raise the concern that chronic NAC therapy may induce similar vascular pathology in patients.
\end{abstract}

\section{A relevant in vivo NO transfer reaction}

Within $\mathrm{rbc}$, the oxygenated (relaxed, Rstructure) and deoxygenated (tight, Tstructure) forms of hemoglobin ( $\mathrm{Hb})$ exist in dynamic equilibrium. Allosteric factors (e.g., $\mathrm{CO}_{2}, \mathrm{pH}$, and 2,3-diphosphoglycerate) profoundly influence the affinity of $\mathrm{Hb}$ for $\mathrm{O}_{2}$ and, hence, this dynamic relationship. The covalent addition of an NO group to the thiol group of cysteine is termed $S$-nitrosylation (1). Within an $\mathrm{rbc}$, the $S$-nitrosothiol modification can occur on small molecular species (e.g., glutathione), soluble proteins (e.g., tetrameric $\mathrm{Hb}$ ), and integral membrane proteins (e.g., anion exchanger AE1 or band 3) (2). NO transfer reactions occurring during erythrocyte deoxygenation may signal hypoxia. As early as 1996, Stamler and colleagues suggested a new wrinkle in the $\mathrm{Hb}$ oxygenation/deoxygenation cycle (3). They argued that a key cysteine residue within the $\beta$-globin chain of $\mathrm{Hb}$ (Cys $\beta 93)$ was biologically relevant to the control of local blood flow. They proposed that $S$-nitroso-hemoglobin (SNO-Hb) transports NO to the microcirculation. The

Nonstandard abbreviations used: GSNO, S-nitrosoglutathione; $\mathrm{Hb}$, hemoglobin; HIF, hypoxia-inducible factor; NAC, $N$-acetylcysteine; $\mathrm{PAH}$, pulmonary arterial hypertension; $\mathrm{pVHL}$, von Hippel-Lindau protein; SNOAC, S-nitroso- $N$-acetylcysteine; SNO-Hb, S-nitroso-hemoglobin.

Conflict of interest: The author has declared that no conflict of interest exists.

Citation for this article: J. Clin. Invest. 117:2377-2380 (2007). doi:10.1172/JCI33136. synthesis of SNO-Hb is favored in the $\mathrm{R}$ structure (present in highly oxygenated blood) and seems to involve the transfer of NO from the iron atom in iron nitrosyl (FeNO) Hb. NO bound to iron complexed with the heme of $\mathrm{Hb}$ is transferred to the thiol group of Cys $\beta 93$ following oxygenation. This newly formed SNO-Cys $\beta 93$ is buried deep within oxygenated $\mathrm{Hb}$. The $\mathrm{O}_{2}-\mathrm{Hb}$ dissociation curve for $\mathrm{SNO}-\mathrm{Hb}$ is left-shifted and is reminiscent of that of fetal $\mathrm{Hb}$ in that SNO-Hb and fetal $\mathrm{Hb}$ have a higher affinity for $\mathrm{O}_{2}$ than adult $\mathrm{Hb}$. As the concentration of $\mathrm{O}_{2}$ decreases, $\mathrm{SNO}-\mathrm{Hb}$ and fetal $\mathrm{Hb}$ are less willing to part with $\mathrm{O}_{2}$. However, in an elegant and physiologically unique fashion, with the allosteric transition of $\mathrm{Hb}$ from the $\mathrm{R}$ state to the $T$ state when oxygen concentrations fall, the $S$-nitrosothiol group on Cys $\beta 93$ is exposed to solvent, and the NO groups are shuttled to other thiols, such as those that reside within glutathione and/or the rbc membrane protein AE1 (Figure 1). Therefore, the release of NO bioactivity from $\mathrm{SNO}-\mathrm{Hb}$ is favored in the $\mathrm{T}$ structure (deoxygenated) form of $\mathrm{Hb}$. It is of great interest for the study of normal physiology that the binding and release of $\mathrm{NO}$ groups from $\mathrm{Hb}$ is synchronized with the binding and release of $\mathrm{O}_{2}(1)$. When viewed from the perspective that blood flow is the principal determinant of $\mathrm{O}_{2}$ delivery, rather than $\mathrm{O}_{2}$-carrying capacity, this newly appreciated pathway helped explain how rbc could induce blood vessels to constrict when tissue $\mathrm{O}_{2}$ concentrations rise but dilate when $\mathrm{O}_{2}$ concen- trations fall $(4,5)$. rbc dynamically match blood flow to metabolic demand, in part by adjusting NO bioavailability (4).

In retrospect, this elegant pathway was too slow in gaining acceptance. In the early days of NO biology, everybody knew that $\mathrm{Hb}$, at least oxygenated $\mathrm{Hb}$, was a potent scavenger of $\mathrm{NO}$ and an inhibitor of endothelium-derived relaxing factor or NO bioactivity. The unappreciated nuance was the in vivo cycling that occurred between the oxygenated and deoxygenated forms of $\mathrm{Hb}$ and the relevance of SNO-Hb. In contrast to the nearly complete saturation of $\mathrm{Hb}$ with oxygen, typically less than $1 \%$ of $\mathrm{Hb}$ molecules contain NO, at least in normal physiology (6). Though chemical, electrochemical, and spectroscopic methods for measuring $S$-nitrosothiol compounds had proven adequate for analysis of in vitro $S$-nitrosothiol chemistry, assessments of the physiological NO-Hb situation would prove to be very challenging (7).

\section{In vivo SNO biology: the importance} of low-molecular-weight species

Low-molecular-weight $S$-nitrosothiols, such as amino acids and small peptides, participate in homeostatic integrative pathways. For example, hypoxia-induced increases in minute ventilation are signaled by SNO-Hb-derived low-molecular-weight $S$-nitrosothiols (8). S-nitrosoglutathione (GSNO), S-nitrosocysteinyl glycine, and $S$-nitroso-L-cysteine, but not $S$-nitroso-Dcysteine, are hypoxia-mimetic and reproduce the ventilatory effects of hypoxia on respiratory centers in the nucleus tractus solitarius of the CNS.

The current work in this issue of the JCI by Palmer et al. (9) started with a pharmacological observation. $\mathrm{N}$-acetylcysteine (NAC) is a pharmacological agent, an $N$-acetyl derivative of the amino acid L-cysteine, that is a commonly used antioxidant. When administered to mice for three weeks at $10 \mathrm{mg} / \mathrm{ml}$ in drinking water, NAC induced pulmonary arterial hypertension (PAH). The authors present 


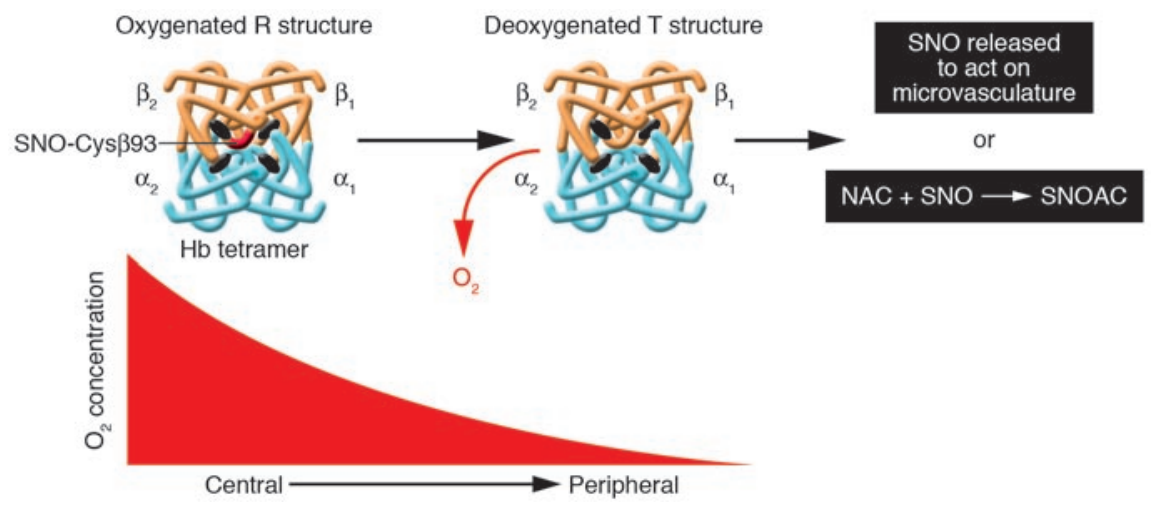

\section{Figure 1}

$S$-nitrosothiols and the $\mathrm{Hb} \mathrm{O}_{2}$ cycle. The synthesis of SNO-Hb is favored in the highly oxygenated $\mathrm{R}$ structure of tetrameric $\mathrm{Hb}$. SNOCys $\beta 93$ is buried deep within oxygenated $\mathrm{Hb}$. $\mathrm{SNO}-\mathrm{Hb}$ transports NO to the microcirculation. Less than $1 \%$ of $\mathrm{Hb}$ carries NO. With a switch in $\mathrm{Hb}$ to the deoxygenated $\mathrm{T}$ structure when $\mathrm{O}_{2}$ concentrations fall, the $S$-nitrosothiol group is exposed and transferred to other thiol acceptors. NAC can serve as a pharmacological bait and forms SNOAC. compelling evidence that $S$-nitroso- $N$ acetylcysteine (SNOAC) is the molecular intermediate formed in vivo that signals from the erythrocyte to the pulmonary tissue, and presumably the vascular endothelium, to cause PAH. The tissue changes were comparable to the effect of chronic normobaric hypoxia. Convincing data are presented indicating that desaturation of $\mathrm{rbc}$ in the periphery leads to SNOAC formation. Furthermore, the authors found that exogenous SNOAC also elicited hypoxia-mimetic toxicity in the pulmonary vasculature. The ex vivo exposure of rbc to sustained hypoxia is accompanied by a buildup of heme FeNO species. Intramolecular transfer of $\mathrm{NO}$ to Cys $\beta 93$ is impaired. This results in a deficiency in the vasodilator SNO-Hb, and $\mathrm{rbc}$ elicit a vasoconstrictor response in isolated vessel preparations. Consistent with this finding, $\mathrm{SNO}-\mathrm{Hb}$ levels are virtually absent in hypoxic patients with moderate to severe PAH (10). Therefore, at least in this disease, the $S$-nitrosothiol recycling pathways are perturbed and are relevant to the patient's phenotype. Palmer et al. also report decreased SNO-Hb levels in the left ventricle of NAC-treated mice (9). One may wonder whether this may be relevant to the observed toxicity of chronic NAC treatment. The authors also note that SNOAC levels fall between the right and left ventricle. Surprisingly, when SNOAC was administered systemically, it did not elicit toxicity in other systemic vascular beds, such as the liver or kidney (9). This issue remains to be fully addressed but may hint at the relevance of the $S$-nitrosothiol recycling pathways, such as seen with $\mathrm{SNO}-\mathrm{Hb}$.

The authors' model is that exogenous, low-molecular-weight $S$-nitrosothiols can cause blood vessel injury (9). The authors refute a competing model. They consid- ered whether chronic NAC treatment could deplete endogenous endothelial cell-derived NO. This would leave the animals functionally NO deficient. Indeed, an acute systemic NO deficit accounts for the acute rise in systemic blood pressure, vasoconstriction, and thromboses that have been associated with Hb-based blood substitutes (11). When considering a model of chronic NO deficit, it is relevant that eNOS $^{-/}$mice also exhibit the phenotype of $\mathrm{PAH}$ and are especially sensitive to further

hypoxic insult. An elegant experimental design allowed Palmer et al. to demonstrate that chronic administration of exogenous SNOAC, but not NAC, could induce disease in $\mathrm{eNOS}^{-/-}$mice (9). The authors appropriately argue that eNOS is key in the biology of the observed NAC response.

\section{SNO on the mountain of HIF biology}

The current work builds upon our understanding of how $S$-nitrosothiols affect hypoxia-inducible factor (HIF) tran-
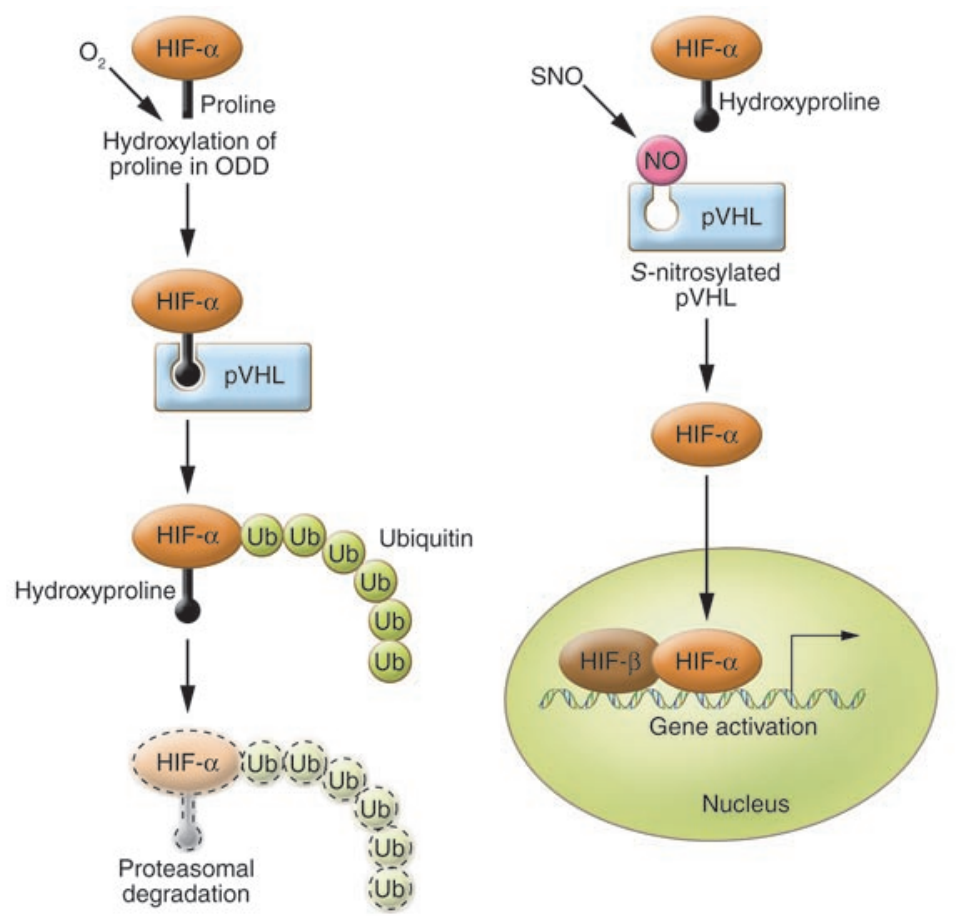

Figure 2

$S$-nitrosylation of $\mathrm{pVHL}$ mimics hypoxia. $\mathrm{pVHL}$ is essential for the ubiquitination and rapid degradation of HIF- $\alpha$. Posttranslational hydroxylation of proline residues in the oxygen-dependent degradation (ODD) domain of HIF- $\alpha$ is required for the interaction between HIF- $\alpha$ and pVHL. This requires molecular oxygen. $\mathrm{pVHL}$ is a target for $S$-nitrosylation. HIF- $\alpha$ and $\mathrm{pVHL}$ fail to interact, and the degradation of HIF- $\alpha$ is impaired. Therefore, even in the presence of oxygen, the cells perceive a hypoxic stimulus. Ub, ubiquitin. 
scription pathways. HIF- $\alpha$ subunits are degraded by the $26 \mathrm{~S}$ proteasomal pathways during normoxia. The von Hippel-Lindau protein ( $\mathrm{pVHL}$ ) is essential for the ubiquitination and rapid degradation of HIF- $\alpha$. Posttranslational hydroxylation of proline residues in the oxygendependent degradation (ODD) domain of HIF- $\alpha$ is required for the interaction between HIF and pVHL (Figure 2). In the setting of hypoxia, this posttranslational modification does not occur, HIF- $\alpha$ is not degraded by the $26 \mathrm{~S}$ proteasomal pathways, and HIFs accumulate. The current work (9) presents compelling evidence that $S$-nitrosothiol-based signaling reactions are hypoxia mimetic and identify pVHL as a target for S-nitrosylation. SNOAC induced $S$-nitrosylation of pVHL in cultured vascular endothelium. Most sporadic renal cell adenocarcinomas are functionally deficient in pVHL, and reconstitution of WT PVHL corrects the malignant phenotype (12). In 786-O renal carcinoma cells stably overexpressing wild-type pVHL, GSNO and SNOAC increased pVHL $S$-nitrosylation (9). The authors argue that cysteine at amino acid position 162, which is known to be important in PVHL biology and commonly mutated in renal carcinoma patients, is the critical residue for $S$-nitrosylation. Though the authors could not exclude $S$-nitrosylation of a target protein that interacts with this pVHL site, the model of parsimony would suggest that $S$-nitrosylation of Cys 162 of pVHL disrupts functional pathways implicated in the ubiquitination and degradation of HIF- $\alpha$ (9). Therefore, taken together, the current results provide important new perspective on how NO can be hypoxia mimetic. pVHL is a target for $S$-nitrosylation, at least in vitro (Figure 2). This insight is novel and adds a further level of understanding of how NO and HIF interact.

This is a complex area for study. Conflicting findings have previously been reported on the effects of NO on HIF biology. Clarity is starting to emerge. The key issue is whether the studies are done under normoxic or hypoxic conditions. Consensus indicates that under normoxic conditions, NO augments HIF activity and is, therefore, hypoxia-mimetic. When cells are truly hypoxic, in contrast, NO inhibits mitochondrial oxygen consumption and is associated with redistribution of oxygen at the subcellular levels (13). The end effect in hypoxic conditions is that HIF activity can be blunted by NO. These in vitro findings underscore the need to address NO biology under normal and hypoxic settings. A similar reasoning could be applied in the current in vivo work. It would be important to more completely address the effect of chronic NAC administration in hypoxic in vivo models.

\section{NAC and pulmonary hypertension}

The current work uses NAC as a bait for $S$-nitrosylation (9). NAC is used clinically as an inhaled mucolytic agent for respiratory disease and an i.v. agent for the treatment of acetaminophen/paracetamol intoxication. In the latter case, preservation of intracellular hepatocyte stores of glutathione reduces drug-induced hepatotoxicity and is the standard of care. Other uses are more controversial. For instance, NAC has been used in the prevention of radiocontrast-induced acute renal failure (14). At present, NAC is not recommended routinely for this indication because clinical trials have given inconsistent results. With these findings as background, how does NAC cause PAH? Some key points need highlighting. To avoid a clinical assessment that was unidimensional, the authors' work addressed multiple time points and considered a variety of NAC concentrations (9). It is key that the NAC-induced effect requires long-term treatment. The chronic administration of GSNO did not induce PAH (9). This is surprising, since GSNO has hypoxia-mimetic effects in vitro. This may suggest that GSNO toxicity is prevented by mechanisms that may be bypassed by SNOAC.

Whether the current findings in murine models are relevant to the utility and/or long-term risk of NAC use in patients warrants debate (9). In normal subjects it can be a hypoxia mimetic - increases in erythropoietin levels and augmented hypoxic ventilatory responses have been described (15). NAC has been used as chronic therapy in disease. For example, when NAC was used chronically as an antioxidant for idiopathic pulmonary fibrosis, there was no clear evidence of pulmonary toxicity (16). Yet such studies in sick patients are complicated in that we do not know whether $S$-nitrosylation biology is normal in these conditions at baseline. In this respect, it is important to distinguish $S$-nitrosothiol formation from $S$-nitrosothiol effects because another key variable, one that is especially relevant in disease, is the regulated expression of NOS isoforms by hypoxia. Messenger RNA expression and enzymatic activity of all three human NOS isoforms, namely neuronal NOS (nNOS, encoded by NOS1), iNOS (NOS2), and eNOS (NOS3), are regulated by hypoxia. For instance, hypoxia leads to decreases in eNOS mRNA and increases in $n N O S$ mRNA expression in vascular endothelium and vascular smooth muscle cells, respectively $(17,18)$.

In summary, NAC induces vascular pathology in the lung that is functionally and morphologically equivalent to that observed with chronic hypoxia (9). These findings are timely for two reasons. First, they begin to address the disconnect that exists between our understanding of $S$-nitrosothiol biology in vitro versus in vivo. We need a more complete understanding of $S$-nitrosothiol biology in vivo. Second, they put the spotlight on an antioxidant drug that is commonly used in clinical medicine. Whether chronic NAC therapy can induce vascular pathology in patients requires scrutiny.

\section{Acknowledgments}

P.A. Marsden is a recipient of a Career Investigator Award from the Heart and Stroke Foundation of Canada and is supported by a grant from the Canadian Institutes of Health Research (CIHR MOP 37778).

Address correspondence to: Philip A. Marsden, Room 7358, Medical Sciences Building, University of Toronto, 1 King's College Circle, Toronto, Ontario M5S 1A8, Canada. Phone: (416) 978-2441; Fax: (416) 978-8765; E-mail: p.marsden@utoronto.ca.

1. Singel, D.J., and Stamler, J.S. 2005. Chemical physiology of blood flow regulation by red blood cells: the role of nitric oxide and S-nitrosohemoglobin. Annu. Rev. Physiol. 67:99-145.

2. Hess, D.T., Matsumoto, A., Kim, S.O., Marshall, H.E., and Stamler, J.S. 2005. Protein S-nitrosylation: purview and parameters. Nat. Rev. Mol. Cell Biol. 6:150-166.

3. Jia, L., Bonaventura, C., Bonaventura, J., and Stamler, J.S. 1996. S-nitrosohaemoglobin: a dynamic activity of blood involved in vascular control. Nature. 380:221-226.

4. Allen, B.W., and Piantadosi, C.A. 2006. How do red blood cells cause hypoxic vasodilation? The SNOhemoglobin paradigm. Am. J. Physiol. Heart Circ. Physiol. 291:H1507-H1512.

5. Foster, M.W., Pawloski, J.R., Singel, D.J., and Stamler, J.S. 2005. Role of circulating S-nitrosothiols in control of blood pressure. Hypertension. 45:15-17.

6. Liu, L., et al. 2004. Essential roles of S-nitrosothiols in vascular homeostasis and endotoxic shock. Cell. 116:617-628.

7. Gow, A., Doctor, A., Mannick, J., and Gaston, B. 2007. S-Nitrosothiol measurements in biological systems. J. Chromatogr. B Analyt. Technol. Biomed. Life Sci. 
851:140-151

8. Lipton, A.J., et al. 2001. S-Nitrosothiols signal the ventilatory response to hypoxia. Nature. 413:171-174.

9. Palmer, L.A., et al. 2007. S-nitrosothiols signal hypoxia-mimetic pathology. J. Clin. Invest. 117:2592-2601. doi:10.1172/JCI29444.

10. McMahon, T.J., et al. 2005. A nitric oxide processing defect of red blood cells created by hypoxia: deficiency of S-nitrosohemoglobin in pulmonary hypertension. Proc. Natl. Acad. Sci. U. S. A. 102:14801-14806.

11. Diesen, D., and Stamler, J.S. 2007. S-Nitrosylation and PEGylation of hemoglobin: toward a blood substitute that recapitulates blood. J. Mol. Cell.
Cardiol. 42:921-923.

12. Kaelin, W.G., Jr. 2002. Molecular basis of the VHL hereditary cancer syndrome. Nat. Rev. Cancer. 2:673-682.

13. Hagen, T., Taylor, C.T., Lam, F., and Moncada, S. 2003. Redistribution of intracellular oxygen in hypoxia by nitric oxide: effect on HIF1alpha. Science. 302:1975-1978.

14. Barrett, B.J., and Parfrey, P.S. 2006. Clinical practice. Preventing nephropathy induced by contrast medium. N. Engl. J. Med. 354:379-386.

15. Hildebrandt, W., Alexander, S., Bartsch, P., and Droge, W. 2002. Effect of N-acetyl-cysteine on the hypoxic ventilatory response and erythropoietin production: linkage between plasma thiol redox state and $\mathrm{O}(2)$ chemosensitivity. Blood. 99:1552-1555.

16. Demedts, M., et al. 2005. High-dose acetylcysteine in idiopathic pulmonary fibrosis. N. Engl. J. Med. 353:2229-2242.

17. Fish, J.E., et al. 2007. Hypoxia-inducible expression of a natural cis-antisense transcript inhibits endothelial nitric-oxide synthase. J. Biol. Chem. 282:15652-15666.

18. Ward, M.E., et al. 2005. Hypoxia induces a functionally significant and translationally efficient neuronal NO synthase mRNA variant. J. Clin. Invest. 115:3128-3139. doi:10.1172/JCI20806.

\title{
$\beta$ Cell transplantation and immunosuppression: can't live with it, can't live without it
}

\author{
Klaus H. Kaestner
}

Department of Genetics and Institute for Diabetes, Obesity, and Metabolism, University of Pennsylvania, Philadelphia, Pennsylvania, USA.

\begin{abstract}
Since the first diabetic was treated with insulin in 1922, millions of patients have relied on frequent insulin injections and glucose monitoring to combat the disease and its complications. Improved immunosuppressive regimens in islet transplantation developed in the Edmonton protocol raised the hopes of diabetics worldwide for a complete cure and insulin independence. However, transplant success has proven to be short-lived and accompanied by significant side effects. Using a clever genetic model for conditional ablation of pancreatic $\beta$ cells in vivo, Nir and colleagues show in this issue of the $J C I$ that the immunosuppressant drugs clinically inhibit $\beta$ cell proliferation in the diabetic setting (see the related article beginning on page 2553). They also demonstrate that $\beta$ cells have a remarkable regenerative capacity and that normal $\beta$ cell mass can recover even in the setting of hyperglycemia. Their new mouse model should aid in the development of improved immunoregulatory strategies and in the elucidation of the molecular pathways that govern $\beta$ cell regeneration.
\end{abstract}

Although insulin therapy for diabetes has come a long way since the hormone's discovery in the 1920s, insulin therapy cannot cure the disease and prevent its devastating complications in the long term. Beginning in the 1970s, whole-organ pancreas transplantation showed that replenishment of the $\beta$ cell complement could fully normalize glucose homeostasis in patients. Transplantation of cadaveric islets into the liver via the portal vein was not successful until 2000, when a glucocorticoid-free immunosuppressive regimen was used and shown to result in insulin independence even in formerly brittle (that is, metabolically unstable) diabetic recipients (1). This advance increased the hopes for diabetics all over the world for a healthy life and led

Conflict of interest: The author has declared that no conflict of interest exists.

Citation for this article: J. Clin. Invest. 117:2380-2382 (2007). doi:10.1172/JCI33375. to dramatic policy changes in biomedical research funding. Because available organ donors would never be sufficient for the treatment of the millions of type 1 diabetics in the world, the NIH and private foundations funded major initiatives to develop new sources of $\beta$ cells for transplantation, for instance through the National Institute of Diabetes and Digestive and Kidney Diseases-funded Beta Cell Biology Consortium (BCBC; http://www.betacell.org).

Multiple sources of cells have been considered as substrates for the development of this cell-based therapy. Among these are embryonic stem cells, hepatocytes, and putative resident endocrine stem cells within the pancreas; yet the success of these efforts has been limited thus far (2). Recent work has refocused the attention of diabetes researchers onto the mature $\beta$ cell itself, because it was shown using genetic lineage tracing that under normal circumstances in healthy rodents, new $\beta$ cells are mostly derived from existing $\beta$ cells (3). Additional support for this concept came from successive labeling of proliferating cells using two thymidine analogs, which showed that adult pancreatic islets do not contain specialized progenitors that turn over rapidly, but rather that most or all $\beta$ cells have the potential to proliferate (4). What these models had not established is whether $\beta$ cell proliferation can also occur in the diabetic setting.

\section{A mouse model for $\beta$ cell regeneration in the diabetic state}

To address this question, Nir and colleagues developed, and describe in this issue of the JCI, a new model for the conditional and controlled ablation of pancreatic $\beta$ cells (5) (Figure 1). Using an elegant genetic trick, they were able to express diphtheria toxin specifically in $\beta$ cells at a time of their choosing, which resulted in the elimination of $\beta$ cells through apoptosis. The treated mice were severely diabetic, consistent with an $80 \%$ reduction in their $\beta$ cell mass and pancreatic insulin content. Unlike commonly used chemical agents employed for the killing of $\beta$ cells such as streptozotocin, there was no bystander effect of the diphtheria toxin, and the degree of $\beta$ cell ablation was reproducible. The beauty of the new system is that it allowed the investigators to record the spontaneous recovery of $\beta$ cell function after ablation by simply removing the inducing agent. Remarkably, within a few weeks after diphtheria toxin expression ceased, $\beta$ cell mass and glucose homeostasis were normalized. This was accompa- 